\title{
Wearable technology in academia: Proposed uses of Google Glass in the life sciences
}

\author{
Bradley Elliott, Artun Sukan \\ University of Westminster
}

\section{Introduction}

For those who have not seen it, heard about it or noticed the fuss about it, Google Glass is a major (indeed, perhaps, the major) entry into the new electronic device category of 'wearable technology'. Glass is a pair of spectacles with a small transparent prism that sits above the right eye and projects information just above the wearer's normal view. The unit also contains hardware similar to that of a basic smartphone, including a forward-facing camera, 16 GB of internal memory, a microphone for voice commands and a small touch-sensitive surface on the right side of the frame, for use when voice control is not sufficient (Figure 1: A).

The features of Glass that we anticipate will make it, or hardware like it, a key tool in learning and teaching environments are: a) the projected personal view; b) the forward-facing camera and c) its ability to understand and respond to spoken commands, allowing the wearer the significant advantage of using it hands-free. This article aims to outline how academic staff in higher education might exploit such a device as this for teaching and learning. We can immediately see four ways in which they might put this new tool to use: as a hands-free computer in a research lab setting; as a video podcast tool to instruct students in complex lab techniques and protocols; as a teaching aid, for lecture notes; for enhanced inclusion of students in a discursive or lecture environment. There have also been proposals, such as for 'flipped classrooms', augmented reality and technique analysis, for how students might use Glass (Dunn, 2013, Parslow, 2014).

Figure 1: A) Example of Google Glass (Wikimedia Commons). B) Example view through Glass, showing an overlay of technique protocol during a western blot.

A)

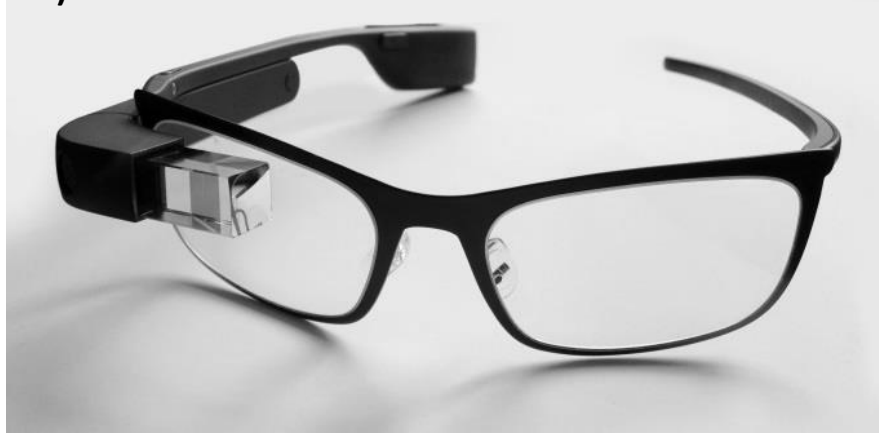

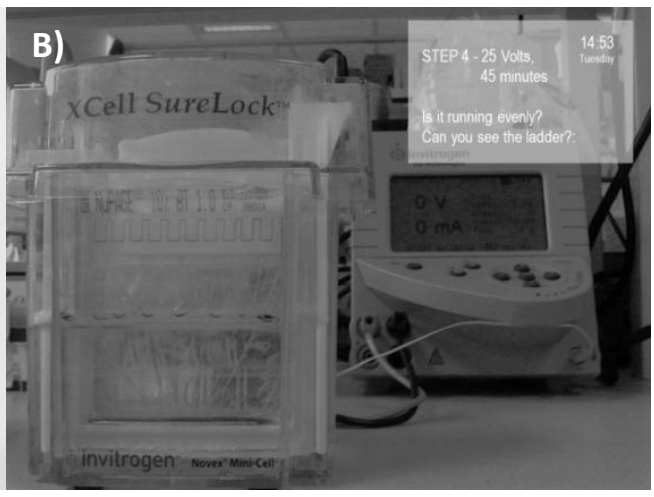

\section{Hands-free device in the teaching and research environment}

Biological science research and teaching necessitates a significant amount of time at the bench. For those who are not familiar with 'bench-top science', there are strict rules and protocols that must be adhered to, to prevent serious harm to researchers and/or damage to their surroundings. Two of these are relevant here: firstly, no phones, no computers, no 
personal electronics; secondly, pens and paper that are in the lab must stay in the lab (i.e. avoid cross-contamination). While this typically precludes the use of modern devices in the biological sciences, there are methods to overcome such barriers. For example, the iPad Project (Dyer, 2012) developed simple disposable plastic covers to allow undergraduate students safely and easily to use iPads as electronic lab books. The ability to perform and check complex dilution calculations, read from electronic instruction sheets or rapidly research a point of interest online without removing gloves and other lab safety equipment is clearly a great advantage (For example, Figure 1: B). There are also numerous occasions during bench-top sciences when the photographic recording of a result or stage is helpful. We therefore propose that a wearable, hands-free screen and camera would be of great use to the research student (or, indeed, to anyone in a research environment) to check protocols, visually record results and access information online.

\section{Laboratory techniques podcast generation}

One continuing issue in the biological sciences is the financial pressure upon the teaching of practical undergraduate sessions. The intended learning outcome for students is typically practical experience in biochemical assays and/or in vitro techniques. These sessions are costly, in both money and time, to the university. Furthermore, there is often a significant difference between the amount of written information given for a protocol and what extra notes are needed to complete a protocol successfully. For example, when describing a common protein quantification technique (the western blot), three lines are typically sufficient. In an undergraduate lab manual for sessions of 50-100 students, this institution uses approximately half a page, whereas, for the instruction of graduate students in a 'oneto-one' lab setting, 1-2 pages of detailed notes, tips and techniques are often required, as such biochemical techniques require the mastery of extensive 'tricks' and skills. We and others (Munch-Harrach et al., 2013) have successfully used podcast-style videos to 'prep' students on complex techniques prior to demonstration or practical sessions. Such audiovisual aids are of great use for delivering rich information and practical skills. Indeed, written protocols often don't or can't include the fine techniques necessary to complete an assay or procedure successfully. We therefore propose that a wearable camera such as Glass makes an excellent tool for the production of first-person podcast videos, overlaid with an information-rich running commentary for the student user, whose hands are thus left free to perform the complex tasks demonstrated.

\section{Seminar and lecture delivery}

The authors of this article don't often use notes when delivering talks, but do occasionally like to have key points or reminders for some presentations. One simple use of a wearable device such as Glass is to couple it to the laptop used for presentations, so that the presenter is able to see projected notes when changing slides; another, even simpler, is for the presenter to use a projected timer/countdown for seminars, brief talks or time-limited conference presentations, when delivery and timing are so critical.

A further use in the lecture setting would be the live recording of lectures as they are delivered; to help their revision, students might well choose to record the spoken part of a lecture or seminar. However, some academics are cautious about audio recording, as words can be misunderstood out of context. Furthermore, recording of inclusive/discursive lectures will include not only the academic's voice, but those of participants also, which may have 
legal implications (JiscLegal, 2010). To overcome this difficulty, an academic might upload a version of the lecture (as it is or edited to avoid potential infringement of spoken word ownership laws) that represents the accepted values of the institution. We therefore propose that an academic wearing such a device could record a delivered lecture or seminar, which recording, in turn, could be attached to slides or notes and uploaded to a virtual learning environment as a more complete resource. While this function does mirror those of more common recording devices currently in use, the pairing of recording technologies offering different key features may simplify the process and not necessarily cause the academic to be overwhelmed by a plethora of tools.

\section{Enhanced student inclusion}

One current use of Glass that demonstrates its potential for enhancing student inclusion is an app called 'Captioning on Glass'. Designed for users who are deaf or have impaired hearing, this app records the spoken words it hears and plays them back to the wearer as caption text in real time. This has allowed deaf users to take part in lectures and even group discussions (Mager, 2014). The eye-level screen allows the user to maintain eye contact during a discussion, rather than having to turn away from the speaker and thus breaking the flow of conversation.

\section{Barriers to overcome}

Any new technology has barriers to be overcome. For Glass, the major concern to date has been one of privacy: some individuals are uncomfortable about the idea of being under the scrutiny of cameras attached to the heads of those around them. Currently, also, cost is an issue, but this will fall as devices become more widely used. Google suggests that battery life is 'all day', but early reviews suggest that three to five hours of constant use may be more realistic, which may be insufficient for longer tasks. In addition, an examination of the tool suggests that several barriers may arise in use. Having a small screen permanently overlaying the right eye may prove more of a hindrance than an advantage in maintaining engagement and may result in a perceived or even physical barrier between learners and teachers. As opposed to collaborative tools, such as 'SMART Board' interactive displays, Glass, as an individual experience, may preclude interactive efforts during group tasks. Being constantly connected to the internet will also allow users to access a range of functions, such as e-mails, social networks and calendar reminders. Since this tool currently requires an unbroken internet connection to perform the majority of its functions, selfdiscipline in its use (just as in the need to remember to silence one's cell phone!) will be essential.

\section{Conclusion}

Like smartphones in the last eight years and tablets in the last four, wearable technologies such as Glass are poised to become a ubiquitous item in society; they have the ability to enhance teaching and learning in higher education. In this article, we have proposed some of their likely practical applications and considered some disadvantages. We also note that, as is the case with any new tool or technological advance, we are likely to see further, perhaps unexpected, ways in which users will apply them within an educational context. 


\section{Reference list}

Dunn, J. 2013. Teachers guide to Google Glass [Online]. Available at http://www.edudemic.com/guides/the-teachers-guide-to-google-glass/. [Accessed 01-022015].

Dyer, P. 2012. iPads in Labs at the University of Greenwich [Online]. University of Greenwich. Available: https://www.youtube.com/watch?v=0BXj56DIGFc [Accessed 13-082014].

JiscLegal 2010. Recording Lectures: Legal Considerations. Jisc online. Available: http://www.jisclegal.ac.uk/Portals/12/Documents/PDFs/Recording\%20Lectures.pdf [Accessed 18-08-2014].

Mager, W. 2014. Live subtitles: How smart technology could help deaf people [Online]. BBC. Available: http://www.bbc.co.uk/news/blogs-ouch-28672182 [Accessed 13-08-2014].

Munch-Harrach, D., Kothe, C. \& Hampe, W. 2013. Audio podcasts in practical courses in biochemistry - cost-efficient e-learning in a well-proven format from radio broadcasting. GMS Z Med Ausbild, 30, 44.

Parslow, G. R. 2014. Commentary: Google Glass: a head-up display to facilitate teaching and learning. Biochem Mol Biol Educ, 42, 91-2. 